\title{
Relationship between Duration of Untreated Psychosis and Intrinsic Corticostriatal Connectivity in Patients with Early Phase Schizophrenia
}

\author{
Deepak K Sarpal', Delbert G Robinson ${ }^{2,3,4}$, Christina Fales ${ }^{2}$, Todd Lencz ${ }^{2,3,4}$, Miklos Argyelan ${ }^{2,3,4}$, \\ Katherine H Karlsgodt ${ }^{5}$, Juan A Gallego ${ }^{6}$, Majnu John ${ }^{2,4}$, John M Kane ${ }^{2,3,4}$, Philip R Szeszko ${ }^{7,8}$ and \\ Anil K Malhotra ${ }^{*, 2,3,4}$
}

\begin{abstract}
'Department of Psychiatry, University of Pittsburgh School of Medicine, Pittsburgh, PA, USA; ${ }^{2}$ Department of Psychiatry, The Zucker Hillside Hospital, Northwell Health, Glen Oaks, NY, USA; ${ }^{3}$ Center for Psychiatric Neuroscience, Feinstein Institute for Medical Research, Manhasset, NY, USA; ${ }^{4}$ Department of Psychiatry, Hofstra Northwell School of Medicine, Hempstead, NY, USA; ${ }^{5}$ Department of Psychology, University of California, Los Angeles, CA, USA; ${ }^{6}$ Department of Psychiatry, Weill Cornell Medical College, New York, NY, USA; ${ }^{7}$ Department of Psychiatry, Icahn School of Medicine at Mount Sinai, New York, NY, USA; ${ }^{8}$ James J. Peters VA Medical Center, Bronx, NY, USA
\end{abstract}

\begin{abstract}
Patients with first-episode psychosis experience psychotic symptoms for a mean of up to 2 years prior to initiation of treatment, and long duration of untreated psychosis (DUP) is associated with poor clinical outcomes. Meanwhile, evidence compiled from numerous studies suggests that longer DUP is not associated with structural brain abnormalities. To date, few studies have examined the relationship between DUP and functional neuroimaging measures. In the present study, we used seed-based resting-state functional connectivity to examine the impact of DUP on corticostriatal circuitry. We included 83 patients with early phase schizophrenia and minimal exposure to antipsychotic drugs ( $<2$ years), who underwent resting state scanning while entering 12 weeks of prospective treatment with secondgeneration antipsychotic drugs. Functional connectivity maps of the striatum were generated and examined in relation to DUP as a covariate. Mediation analyses were performed on a composite measure of corticostriatal connectivity derived from the significant results of our DUP analysis. We found that longer DUP correlated with worse response to treatment as well as overall decreased functional connectivity between striatal nodes and specific regions within frontal and parietal cortices. Moreover, the relationship between DUP and treatment response was significantly mediated by corticostriatal connectivity. Our results indicate that variation in corticostriatal circuitry may play a role in the relationship between longer DUP and worsened response to treatment. Future prospective studies are necessary to further characterize potential causal links between DUP, striatal circuitry and clinical outcomes.

Neuropsychopharmacology (2017) 42, 22I4-222I; doi:I0.I038/npp.2017.55; published online 5 April 2017
\end{abstract}

\section{INTRODUCTION}

Individuals experiencing their first-episode of psychosis interface with psychiatric treatment after a mean of up to 2 years of untreated psychotic symptoms (Loebel et al, 1992; Robinson et al, 2015). Longer duration of untreated psychosis (DUP), a clinical variable marking the amount of time psychotic symptoms exist prior to treatment with antipsychotic drugs, is linked with a number of diseaserelated outcomes. In particular, longer DUP has been associated with increased severity of negative symptoms (Boonstra et al, 2012; Penttilä et al, 2014; Perkins et al, 2004), worse functional and social outcomes (Marshall et al, 2005;

*Correspondence: Dr AK Malhotra, Division of Psychiatry Research, Department of Psychiatry, The Zucker Hillside Hospital, 75-59 263rd Street, Glen Oaks, NY I I004, USA, Tel: 7 I8 470 80 I2, Fax: 718343 |659, E-mail: amalhotra@northwell.edu

Received I8 September 2016; revised 31 January 2017; accepted II February 2017; accepted article preview online 15 March 2017
Penttilä et al, 2014), and overall increased global psychopathology (Perkins et al, 2004). Psychotic symptoms themselves may be more refractory to treatment with a prolonged DUP, given a consistent modest association with poorer response to antipsychotic drug treatment (GunduzBruce et al, 2005; Loebel et al, 1992; Perkins et al, 2005; Robinson et al, 2004). While these observations have prompted a call for systems-level initiatives to decrease DUP (Addington et al, 2015; Cotter et al, 2016; Srihari et al, 2014), the neurobiological impact of untreated psychosis remains unknown.

Clinical findings relating DUP to worse outcomes also gave rise to the possibility that untreated psychotic symptoms might impact neurophysiology (Wyatt, 1991). While this idea remains debated (Rund, 2014), structural neuroimaging and neurocognitive studies have examined effects related to length of DUP. A few reports have put forth evidence for reduced gray matter in regions within planum temporale (Takahashi et al, 2007), basal ganglia (CrespoFacorro et al, 2007), parietal and frontal lobes (Malla et al, 
2002). However, larger studies report no significant relationship between DUP and measures of brain structure (Ho et al, 2003, 2005; Rund, 2014). This was supported by a recent review that reports no collective agreement across structural imaging studies (Anderson et al, 2015). Similarly, neurocognitive studies have not definitively associated DUP with neurocognitive abnormalities (Goldberg et al, 2009). Meanwhile, functional neuroimaging has not been extensively investigated in relation to DUP, and to date no study has directly examined effects on intrinsic functional connectivity.

In our previous work, we focused on resting-state functional connectivity of the striatum as a treatmentrelated biomarker in patients with first-episode schizophrenia. Efficacious treatment of psychotic symptoms was associated with increased connectivity between striatum and frontal and limbic regions (Sarpal et al, 2015). In addition, an index of functional interactions of the striatum showed promise as a prognostic biomarker in patients with first-episode schizophrenia and chronic patients with psychotic disorders (Sarpal et al, 2016). In light of its role in treatment response, and evidence for altered striatal functioning in individuals at high risk for transition to psychotic states (Dandash et al, 2014; Howes et al, 2011), the examination of striatal functional connectivity to the relationship between DUP and treatment response holds considerable promise.

The goal of the present study was to examine the relationship between functional connectivity of the striatum, DUP, and treatment outcome. Following the methods of our previous studies, we generated functional connectivity maps of the striatum and performed voxel-wise correlational analyses with DUP in a cohort of patients in their early phase of a schizophrenia spectrum disorder, with $<2$ years of exposure to antipsychotic medication. Patients underwent scanning while starting prospective treatment with antipsychotic drugs and were followed for 12 weeks of treatment. We tested the hypothesis that that an index of corticostriatal connectivity mediates the relationship between longer DUP and worsened response to treatment.

\section{MATERIALS AND METHODS}

\section{Participants}

All patients $(N=83)$ included in the present study were between the ages of 15 and 40, experiencing psychosis with minimal prior treatment, and scanned with resting-state fMRI while starting antipsychotic treatment as per NIMHfunded clinical trials. Patients were diagnosed with a schizophrenia-spectrum disorder, including schizophrenia $(N=57$, schizophreniform disorder $(N=18)$, schizoaffective disorder $(N=2)$, or psychotic disorder, not otherwise specified $(N=6)$. Diagnoses were based on the Structured Clinical Interview for Axis I Diagnostic and Statistical Manual-IV Disorders (SCID), supplemented by information from clinicians and family members. Written informed consent (or assent with written parental/guardian consent for those under age 18 years) was obtained as per a protocol approved by the Institutional Review Board of Northwell Health. Additional details regarding exclusion criteria for our study participants are available in the Supplementary Materials and Methods.
Patients from two independent cohorts were included in the study. In cohort 1, patients were treated in a doubleblind, randomized controlled trial with either risperidone (dose range: $1-6 \mathrm{mg}$ ) or aripiprazole $(5-30 \mathrm{mg}$ ) for 12 weeks (R01MH060004; ClinicalTrials.gov ID: NCT00320671). In cohort 2, all patients were treated with risperidone (dose range $1-6 \mathrm{mg}$ ) plus randomized adjunctive, double-blind treatment with either an omega-3-fatty acid supplement (740 mg eicosapentaenoic acid, $400 \mathrm{mg}$ docosahexaenoic acid, and $4 \mathrm{mg} / \mathrm{g}$ Tocopherol) or placebo for 12 weeks (R21MH101746; ClinicalTrials.gov ID: NCT01786239). Cohort 1 had at most 2 weeks of prior treatment, while cohort 2 had up to 2 years of treatment. Details regarding additional medications allowed in our patients are available in the Supplementary Materials and Methods.

Clinical ratings were administered weekly for the first month, and then every 2 weeks thereafter until week 12 . Psychopathology was rated with the Brief Psychiatric Rating Scale-Anchored version (BPRS-A) and the Clinical Global Impressions Scale (CGI). Consistent with our prior studies (Robinson et al, 2015; Sarpal et al, 2016), stringent treatment response criteria were defined, a priori. Patients were categorized as responders if they had two consecutive, sustained ratings of a CGI improvement score of much or very much improved, as well as a rating of 3 or less on psychosis-related items of the BPRS-A (conceptual disorganization, grandiosity, hallucinatory behavior, and unusual thought content).

We defined DUP as the time from the emergence of psychotic symptoms to initiation of antipsychotic treatment. Sources for the DUP assessment were the participant, their family and clinical records when available. DUP was calculated and quantified in weeks. Given that DUP is traditionally right-skewed with more individuals presenting with lower durations (Perkins et al, 2005), we performed a common log-transformation of our DUP data to reduce skewing. In all analyses described herein, this log-transformed DUP was used as a continuous variable.

\section{Resting State fMRI Image Acquisition}

All fMRI exams were conducted on a 3T scanner (GE Signa HDx, Manhasset, NY, USA). As in our prior work (Sarpal et al, 2016; Sarpal et al, 2015), we acquired anatomical scans for image registration in the coronal plane using an inversion-recovery prepared $3 \mathrm{D}$ fast spoiled gradient sequence $(\mathrm{TR}=7.5 \mathrm{~ms}, \mathrm{TE}=3 \mathrm{~ms}, \mathrm{TI}=650 \mathrm{~ms}$ matrix $=256 \times$ 256 , FOV $=240 \mathrm{~mm}$ ) producing 216 contiguous images with slice thickness $=1 \mathrm{~mm}$. Our resting-state functional scan lasted $5 \mathrm{~min}$ in duration. The resting state scan included 150 echo-planar imaging (EPI) volumes with a $\mathrm{TR}=2000 \mathrm{~ms}, \mathrm{TE}=30 \mathrm{~ms}$, matrix $=64 \times 64, \mathrm{FOV}=240 \mathrm{~mm}$, slice thickness $=3 \mathrm{~mm}$, and 40 continuous axial oblique slices (one voxel $=3.75 \times 3.75 \times 3 \mathrm{~mm}$ ). During resting state scanning, participants were asked to close their eyes and instructed not to think of anything in particular.

\section{Image Analysis and Preprocessing}

We used FSL (http://www.fmrib.ox.ac.uk) for preprocessing of resting-state scans. The first four EPI volumes were discarded. Each participant's functional image was registered 
to a corresponding structural $\mathrm{T} 1$ image using a linear transformation with six degrees of freedom. This structural image was normalized by a 12-parameter affine transformation to MNI152 space. The combination of these transformations was then applied to each individual's functional dataset. Rigid body motion correction was performed with MCFLIRT and skull stripping was performed with BET. Images were spatially smoothed with a 5-mm FWHM Gaussian kernel. The resulting time series was then high pass filtered at $0.01 \mathrm{~Hz}$. White matter (WM) and cerebrospinal fluid (CSF) masks were generated using FAST by segmentation of each individual's structural image. Mean signal within these masks was extracted. For removal of nuisance variables, each individual's $4 \mathrm{D}$ time series the data were regressed with eight predictors in a general linear model: WM, CSF, and six motion parameters. To avoid interference with our connectivity measures, the global mean was not included in this calculation.

\section{Functional Connectivity Analyses}

To test the functional connectivity of subregions of the striatum within the putamen, caudate nucleus, and nucleus accumbens, we used a seed-based approach. We utilized methods described in our previous work (Sarpal et al, 2016; Sarpal et al, 2015), adopted from Di Martino et al, 2008. Central coordinates of regions of interest (ROIs) were used to create $4 \times 4 \times 4 \mathrm{~mm}$ spheres defined, bilaterally, in: dorsal caudate (DC) $(x= \pm 13, y=15, z=9)$, ventral caudate (VSS) $(x= \pm 10, y=15, z=0)$, ventral caudate/nucleus accumbens (VSI) $(x= \pm 9, y=9, z=-8)$, dorsal rostral putamen (DRP) $(x= \pm 25, y=8, z=6)$, dorsal caudal putamen (DCP) $(x= \pm 28, y=1, z=3)$, and ventral rostral putamen (VRP) $(x= \pm 20, y=12, z=-3)$.

Once ROIs were defined, functional connectivity analyses were conducted. At the first-level, for each patient, scans were used to create correlation maps for each participant for all 12 of our ROIs. Mean activity time courses were extracted from each seed region. Whole-brain voxel-wise correlation maps for each ROI were then created with the extracted waveform as a reference. The resulting correlation maps were Z-transformed.

To account for motion-related artifact in our data, we first eliminated patients with extreme motion (defined by at least
2 s.d. above the average FD) prior to group-level analyses. Mean frame-wise displacement (FD) was calculated in FSL and was also included to control for the residual effect of head motion. Given that use of the data scrubbing to eliminate motion-related artifact offers little advantage over group-level corrections and can correct the data incompletely (Yan et al, 2013), we accounted for head motion at the group-level by including mean FD as a nuisance covariate (Power et al, 2015; Yan et al, 2013).

Group level analyses were performed independently for each ROI in FSL's FLAME. For each ROI, all maps were entered into a general linear model with age, sex, and FD as covariates. Significance was defined voxel-wise at $p<0.005$, with cluster correction at $p<0.05$. All neuroimaging findings reported below met this threshold.

\section{Post Hoc and Mediation Analyses}

All post hoc and mediation analyses were performed in the $\mathrm{R}$ statistical environment (http://www.r-project.org). Raw connectivity values were extracted for all significant clusters using a $3-\mathrm{mm}$ radius around the peak voxel, and entered into a matrix with 83 rows (one for each patient), and connectivity values in columns. For purposes of the data reduction, a principal component analysis was performed on these extracted values to identify explanatory factors within our results. Horn's parallel analysis was performed on our principal components to empirically identify the number of factors to retain for further analyses. Subsequently, we performed a mediation analysis to examine whether retained principal components capture the explanatory variance mediated the relationship between DUP and treatment response. Following the steps by (Judd and Kenny, 1981), we tested: (a) whether the effect of DUP on treatment response is significant in a survival analysis; (b) a significant relationship exists between DUP and our corticostriatal connectivity index; (c) that the relationship between our index and treatment response is significant in a survival analysis; and (d) the direct effect of DUP on treatment response adjusted for our index is non-significant. Mediation effects were defined if all four of these steps were passed (Baron and Kenny, 1986). Aalen's additive hazards model regression analysis was used as our method for examining our survival data with time to response in weeks and

Table I Clinical and Demographic Information

\begin{tabular}{|c|c|c|c|c|c|c|c|c|}
\hline \multirow[t]{2}{*}{ Characteristic } & \multicolumn{3}{|c|}{ Responders $(N=48)$} & \multicolumn{3}{|c|}{ Non-responders $(N=35)$} & \multirow[b]{2}{*}{$t / \chi^{2}$} & \multirow[b]{2}{*}{$P$-value } \\
\hline & Mean & SD & $\mathbf{N}$ & Mean & SD & $\mathbf{N}$ & & \\
\hline Age (years) & 21.9 & 6.0 & & 21.2 & 3.7 & & $t=-0.6(8 I)$ & 0.55 \\
\hline \multicolumn{9}{|l|}{ Sex } \\
\hline Males & & & 35 & & & 26 & $\chi^{2}(1)=0.86$ & 1.0 \\
\hline Females & & & 14 & & & 10 & & \\
\hline Years of education & 12.2 & 2.0 & & 12.3 & 2.4 & & $t=0.22(81)$ & 0.82 \\
\hline Baseline BPRS (total) & 42.3 & 7.6 & & 43.1 & 7.3 & & $t=0.47(81)$ & 0.64 \\
\hline Baseline BPRS (psychotic symptoms) & 4.5 & 3.2 & & 14.0 & 2.9 & & $t=0.99(81)$ & 0.34 \\
\hline
\end{tabular}

Neuropsychopharmacology 


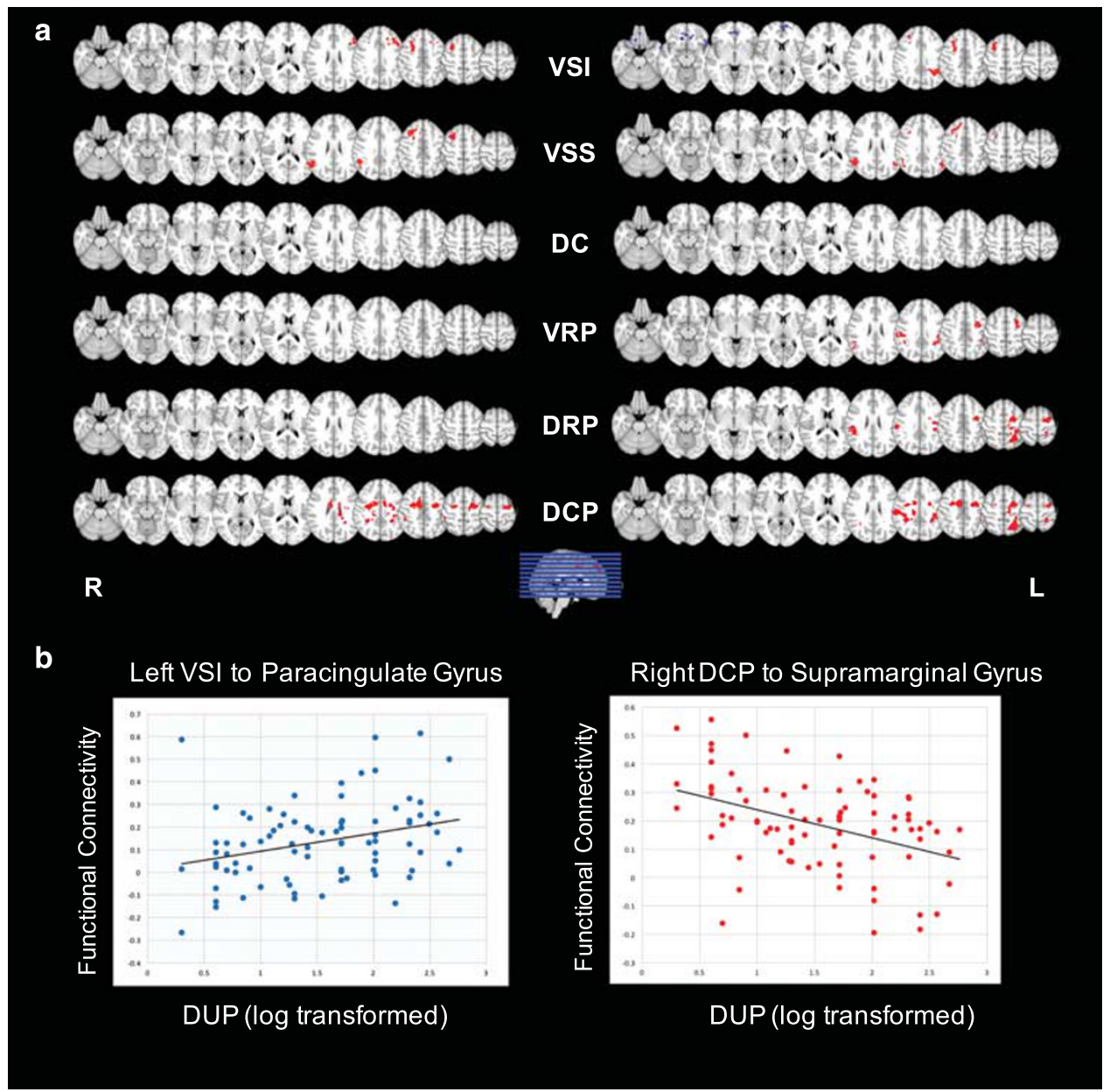

Figure I Functional connectivity results. Whole-brain results of functional connectivity analyses between duration of untreated psychosis and all 12 regions of interest are displayed in (a). In (b), representative connections are plotted, including the left dorsal caudal putamen with a region within left supramarginal gyrus, and the left ventral caudate with an area in the left dorsolateral prefrontal cortex. All results are displayed at $p<0.05$, cluster corrected.

response status as variables. Aalen's additive hazards model is a non-parametric method for analyzing the survival data. It is useful for modeling the relationship between risk factors and time-dependent outcomes.

\section{RESULTS}

\section{Demographics and the Clinical Data}

We included 83 patients in this analysis, 47 from cohort 1 and 36 from cohort 2 (see methods above). Demographic variables are summarized in Table 1. Age, sex, education, and baseline scores of psychopathology ratings were not associated with DUP, and did not differ between responders $(N=48)$ and non-responders $(N=35)$ to treatment. Similarly, no differences were observed in any demographic variables between patients in cohorts 1 and 2. A histogram of our raw DUP data, displayed in Supplementary Figure S1, was right-skewed, consistent with typical distributions. The average DUP across all patients was 102 weeks $(\mathrm{SD}=77$ weeks), and the median was 35 weeks. Common log transformation of these data reduced skewing (Table 1). Patients included in the analyses had a median of 5 days of antipsychotic medication exposure prior to scanning (mean
15 , SD 59 days), and 31 patients were naive to antipsychotic treatment at time of scanning (37\%). We found no relationship between DUP and amount of antipsychotic exposure.

Our response rate to antipsychotic treatment was 56\% during our 12-week medication trial. Longer DUP prior to treatment was associated with poorer rates of response in a survival analysis with time to response and response status as survival parameters ( $p=0.03$; Supplementary Figure S2).

\section{Functional Connectivity}

At the group level functional connectivity maps for each seed region showed consistent connections with maps from prior studies (Di Martino et al, 2008; Sarpal et al, 2016; Sarpal et al, 2015). Consistent with our a priori hypothesis of a negative relationship between DUP and corticostriatal connectivity, we discovered a total of 24 clusters across our 12 seed regions that predominantly showed significantly lower functional connectivity with greater DUP. These significant clusters are displayed in Figure 1 and peak voxels for each cluster are listed with statistical details in Supplementary Table S1. Across these clusters, we observed decreased functional connectivity between striatum and cortex that overlapped in several regions, including the supramarginal gyrus (38\% of 
significant clusters), middle frontal gyrus (17\% of clusters), and cingulate gyrus (12\%). Conversely, we observed a significant positive relationship between increasing DUP and functional connectivity between the ventral striatum/ nucleus accumbens and the subcallosal cortex/orbitofrontal cortex, and medial frontal pole (Figure 1 and Supplementary Table S1).

We extracted connectivity values from our significant clusters and performed several post hoc analyses. No significant differences in connectivity values were found between our patient cohorts. No relationship was found between prior med exposure and connectivity. Patients in our aripiprazole $v s$ risperidone-treated cohort showed no differences in functional connectivity across our significant clusters. Similarly, in cohort 2, placebo versus omega 3 fatty acid-treated patients showed no differences in functional connectivity with significant clusters.

\section{Mediation Analysis}

We further examined our connectivity results in relation to treatment response. The data reduction via principal component analysis over the 24 significant clusters revealed two significant factor that stood out that explained 34 and $9 \%$ of the variance, respectively, in our results; other factors each accounted for $<8 \%$ of the variance. The first principal component significantly predicted treatment response in a survival analysis $(P=0.02)$. We performed an exploratory mediation analysis to test whether this factor mediates the relationship between increased DUP and worsened treatment response (Figure 2). When the first principal component is included as a mediator into the model, the direct path from DUP to treatment response described above $(\beta=-0.18, P=0.03)$ is no longer significant $(\beta=-0.03$, $P=0.07)$. Thus, we found a significant mediation effect, indicating that DUP exerts its effects on treatment response through a variable representing our corticostriatal connectivity results. The second principal component did not significantly relate to treatment response, and was therefore was not a mediator.

\section{DISCUSSION}

In the present study, we report that length of DUP is associated with variation in striatal functional connectivity, and that this variation plays a mediating role in the negative

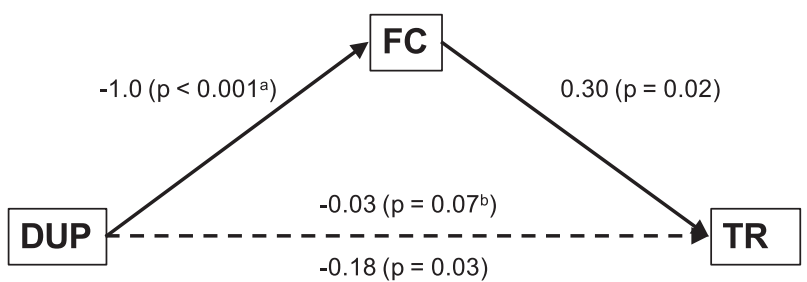

Figure 2 Mediation effect of striatal functional connectivity. ${ }^{a}=$ calculated in functional connectivity analysis; ${ }^{b}=$ DUP is non-significant when adjusted with $F C$ included in the regression. $T R=$ treatment response, DUP $=\log$ transformed duration of untreated psychosis, $F C=$ summation of loadings across all significant results of the first principal component. Beta values are median of beta across all time points in an Aalen's additive hazards model. relationship between DUP and response to treatment. Our analyses included 83 patients with early-phase schizophrenia, pooled from two separate cohorts. Patients had minimal prior antipsychotic exposure, were scanned with resting state fMRI at time of initiation of controlled treatment with a second-generation antipsychotic medication, and were clinically monitored for 12 weeks with stringent a priori treatment response criteria. Consistent with our hypothesis, longer DUP was associated with decreased corticostriatal functional connectivity. We observed an overall negative relationship between DUP and connectivity of the striatum with a group of cortical regions, inclusive of the central executive and dorsal attention networks. The first principal component derived from these results showed a mediating effect on the link between DUP and treatment response.

To our knowledge, this is the first study to directly examine the relationship between DUP and intrinsic connectivity of the striatum, a structure that is repeatedly implicated in the pathophysiology of schizophrenia. Studies have consistently shown, via positron emission tomography, that patients with schizophrenia exhibit increased postsynaptic dopaminergic activity (Abi-Dargham et al, 1998; Howes et al, 2012). More recent work has used fMRI-based activation and connectivity to characterize the role of the striatum in schizophrenia-related pathology. Activation of the striatum during reward processing has been associated with antipsychotic treatment outcome (Nielsen et al, 2012), as well as antipsychotic-induced weight gain (Nielsen et al, 2016). Pretreatment findings have demonstrated abnormal striatal dopamine and connectivity in individuals at risk for chronic psychotic disorders (Howes et al, 2009), as well as decreased caudate volume with longer untreated symptoms in first-episode patients (Crespo-Facorro et al, 2007). The present study adds to this literature by linking DUP to striatal functional connectivity. Future work is necessary to fully characterize our findings with other components of cortico-striato-thalamo-cortical circuits (Alexander et al, 1986).

The present study also adds to a growing literature that implicates the striatum in treatment-related neurobiology. Prior work has shown that functional connectivity of the striatum plays a key role in the mechanism of treatment response in longitudinal analyses (Anticevic et al, 2015; Lahti et al, 2003; Sarpal et al, 2015), and may be used as a prognostic biomarker (Lahti et al, 2009; Sarpal et al, 2016). Here we extend this work by showing that the negative relationship between DUP and treatment response to antipsychotic drugs occurs through differences in striatal interactions. While considering the debate whether untreated psychosis impacts neurophysiology (Rund, 2014; Wyatt, 1991), it is important to emphasize that our results do not establish a causal link between untreated psychosis and deleterious effects on striatal neurophysiology. Further prospective work characterizing the prodromal period is needed to establish causality.

Many of the striatal connections associated with DUP that we describe here map onto predetermined functional networks, including the central executive network. Some of these areas include the middle frontal gyrus, superior frontal gyrus, the anterior cingulate, and the supramarginal gyrus (Seeley et al, 2007). This network of structures has been linked to functions related to manipulation and maintenance 
of information, goal-directed behavior, and problem solving (Miller and Cohen, 2001; Minzenberg et al, 2009; Müller and Knight, 2006). Interestingly, while some studies have shown a relationship between neurocognitive deficit and longer DUP (Gaynor et al, 2009), prior work has also found evidence for preserved cognition in patients with longer DUP (Goldberg et al, 2009; Norman et al, 2001). It remains unknown how our findings relate to executive functioning and other cognitive domains, and whether our findings of reduced executive-striatal connectivity represent a traitrelated or adaptive mechanism. Similarly, we observe a negative association between longer DUP and striatal connectivity with the supramarginal gyrus, a structure located within the inferior parietal lobule. Interestingly, we previously found that increased connectivity between this region and the striatum coincides with less efficacious outcomes after 12 weeks of antipsychotic treatment (Sarpal et al, 2015). Future studies may reconcile these two findings by testing whether DUP results in decreased striatalsupramarginal gyrus connectivity to a degree that impedes an efficacious response to treatment. Additionally, as a key component for language processing (Binder et al, 1997), our results in the supramarginal gyrus may represent a breakdown in language-related processes secondary to worsening psychosis.

Similar to our findings in the central executive network, we also report a finding with a region of the superior frontal gyrus that is partially within the frontal eye field, a component of the dorsal attention network (Corbetta et al, 2008). Prior studies have demonstrated that untreated patients with first-episode schizophrenia show significantly enhanced rapid gaze shifting, suggesting reduced inhibitory control of saccades, a finding that is normalized with antipsychotic treatment (Reilly et al, 2005). Further studies may determine whether DUP may play a role in this normalizing effect.

Combined with our findings in the central executive network, these results highlight that variation in striatal interactions with multiple functional networks is associated with length of DUP. Overall, it is unclear whether our functional connectivity findings are a result of untreated psychosis, or if they represent a trait-related marker present in patients with longer DUP. It is also unclear whether the striatum is primarily involved, or if the results we find are secondary to abnormalities in other neural systems.

There are several limitations to our study. For one, we characterized DUP, often a difficult clinical variable to delineate, in a retrospective manner. Because of this, we are not able to precisely tease out the emergence of frank psychotic symptoms from sub-threshold prodromal symptoms. Future cohort studies are necessary with a prospective and longitudinal design, beginning in the prodromal period and extending through the conversion to illness. Secondly, our study examined the relationship between DUP and a single neuroimaging modality. Further studies are necessary that incorporate multimodal analyses of brain structure and function to better characterize the potential impact of untreated psychosis on neural systems. Thirdly, our analysis includes patients from two separate cohorts, each with 2 treatment arms. While we observe no effect of any one particular subgroup of patients, future studies may focus on a more homogeneous population of patients. Additional studies are also needed to examine whether our findings represent a trait-based marker or a subtype of patients.

To conclude, we report an associative role for functional connectivity of the striatum with DUP and its relationship to treatment response. Longer DUP is associated with an overall decrease in connectivity between striatal nodes and regions within central executive and dorsal attention networks. The first principal component explaining these results shows evidence as a mediator between the negative relationship between DUP and response to treatment. Subsequent studies focused on this question from a prospective and longitudinal approach may be helpful in examining whether abnormal striatal functioning is a consequence of untreated psychosis and causally related to worsened treatment outcomes.

\section{FUNDING AND DISCLOSURE}

This work was Supported by NIMH grants P50MH080173 to Dr Malhotra; P30MH090590 to Dr Kane; R01MH060004, R21MH101746 to Dr Robinson; K23MH100264 to Dr Gallego; and R01MH076995, R21MH101746 to Dr Szeszko. Dr Robinson has been a consultant to Asubio and Shire, and he has received grants from Bristol-Meyers Squibb, Janssen, and Otsuka. Dr Lencz has been a consultant to Eli Lilly. Dr Kane has been a consultant for or received honoraria from Alkermes, Eli Lilly, EnVivo Pharmaceuticals (Forum), Forest (Allergan), Genentech, $\mathrm{H}$ Lundbeck. Intracellular Therapeutics, Janssen Pharmaceutica, Johnson and Johnson, Otsuka, Reviva, Roche, Sunovion and Teva; has received grant support from Otsuka and Janssen; and is a Shareholder in MedAvante, Inc., Vanguard Research Group and LB Pharmaceuticals, Inc. Dr Malhotra is a consultant to Genomind, inc., Takeda, and Forum Pharmaceuticals. Dr Sarpal has received research support from Janssen. The remaining authors declare no conflict of interest.

\section{ACKNOWLEDGMENTS}

We acknowledge our patients and their families, as well as our psychiatry research support staff.

\section{REFERENCES}

Abi-Dargham A, Gil R, Krystal J, Baldwin RM, Seibyl JP, Bowers M et al (1998). Increased striatal dopamine transmission in schizophrenia: confirmation in a second cohort. Am J Psychiatry 155: 761-767.

Addington J, Heinssen RK, Robinson DG, Schooler NR, Marcy P, Brunette MF et al (2015). Duration of untreated psychosis in community treatment settings in the United States. Psychiatr Serv Wash DC 66: 753-756.

Alexander GE, DeLong MR, Strick PL (1986). Parallel organization of functionally segregated circuits linking basal ganglia and cortex. Annu Rev Neurosci 9: 357-381.

Anderson KK, Rodrigues M, Mann K, Voineskos A, Mulsant BH, George TP et al (2015). Minimal evidence that untreated psychosis damages brain structures: a systematic review. Schizophr Res 162: 222-233.

Anticevic A, Hu X, Xiao Y, Hu J, Li F, Bi F et al (2015). Early-course unmedicated schizophrenia patients exhibit elevated prefrontal connectivity associated with longitudinal change. J Neurosci Off J Soc Neurosci 35: 267-286. 
Baron RM, Kenny DA (1986). The moderator-mediator variable distinction in social psychological research: conceptual, strategic, and statistical considerations. J Pers Soc Psychol 51: 1173-1182.

Binder JR, Frost JA, Hammeke TA, Cox RW, Rao SM, Prieto T (1997). Human brain language areas identified by functional magnetic resonance imaging. J Neurosci 17: 353-362.

Boonstra N, Klaassen R, Sytema S, Marshall M, De Haan L, Wunderink L et al (2012). Duration of untreated psychosis and negative symptoms-a systematic review and meta-analysis of individual patient data. Schizophr Res 142: 12-19.

Corbetta M, Patel G, Shulman GL (2008). The reorienting system of the human brain: from environment to theory of mind. Neuron 58: 306-324.

Cotter J, Zabel E, French P, Yung AR (2016). Prolonged duration of untreated psychosis: a problem that needs addressing. Early Interv Psychiatry (doi:10.1111/eip.12308; e-pub ahead of print).

Crespo-Facorro B, Roiz-Santiáñez R, Pelayo-Terán JM, GonzálezBlanch C, Pérez-Iglesias R, Gutiérrez A et al (2007). Caudate nucleus volume and its clinical and cognitive correlations in first episode schizophrenia. Schizophr Res 91: 87-96.

Dandash O, Fornito A, Lee J, Keefe RSE, Chee MWL, Adcock RA et al (2014). Altered striatal functional connectivity in subjects with an at-risk mental state for psychosis. Schizophr Bull 40: 904-913.

Di Martino A, Scheres A, Margulies DS, Kelly AMC, Uddin LQ, Shehzad Z et al (2008). Functional connectivity of human striatum: a resting state FMRI study. Cereb Cortex N Y N 1991 18: 2735-2747.

Gaynor K, Dooley B, Lawlor E, Lawoyin LR, O'Callaghan E (2009). Cognitive deterioration and duration of untreated psychosis. Early Interv Psychiatry 3: 157-160.

Goldberg TE, Burdick KE, McCormack J, Napolitano B, Patel RC, Sevy SM et al (2009). Lack of an inverse relationship between duration of untreated psychosis and cognitive function in first episode schizophrenia. Schizophr Res 107: 262-266.

Gunduz-Bruce H, McMeniman M, Robinson DG, Woerner MG, Kane JM, Schooler NR et al (2005). Duration of untreated psychosis and time to treatment response for delusions and hallucinations. Am J Psychiatry 162: 1966-1969.

Ho BC, Alicata D, Mola C, Andreasen NC (2005). Hippocampus volume and treatment delays in first-episode schizophrenia. Am J Psychiatry 162: 1527-1529.

Ho BC, Alicata D, Ward J, Moser DJ, O'Leary DS, Arndt S et al (2003). Untreated initial psychosis: relation to cognitive deficits and brain morphology in first-episode schizophrenia. Am J Psychiatry 160: 142-148.

Howes OD, Bose SK, Turkheimer F, Valli I, Egerton A, Valmaggia LR et al (2011). Dopamine synthesis capacity before onset of psychosis: a prospective [18F]-DOPA PET imaging study. Am J Psychiatry 168: 1311-1317.

Howes OD, Montgomery AJ, Asselin M-C, Murray RM, Valli I, Tabraham $\mathrm{P}$ et al (2009). Elevated striatal dopamine function linked to prodromal signs of schizophrenia. Arch Gen Psychiatry 66: $13-20$.

Howes OD, Kambeitz J, Kim E, Stahl D, Slifstein M, Abi-Dargham A et al (2012). The nature of dopamine dysfunction in schizophrenia and what this means for treatment: Meta-analysis of imaging studies. Arch Gen Psychiatry 69: 776-786.

Judd CM, Kenny DA (1981). Process analysis estimating mediation in treatment evaluations. Eval Rev 5: 602-619.

Lahti AC, Holcomb HH, Weiler MA, Medoff DR, Tamminga CA (2003). Functional effects of antipsychotic drugs: comparing clozapine with haloperidol. Biol Psychiatry 53: 601-608.

Lahti AC, Weiler MA, Holcomb HH, Tamminga CA, Cropsey KL (2009). Modulation of limbic circuitry predicts treatment response to antipsychotic medication: a functional imaging study in schizophrenia. Neuropsychopharmacol 34: 2675-2690.
Loebel AD, Lieberman JA, Alvir JM, Mayerhoff DI, Geisler SH, Szymanski SR (1992). Duration of psychosis and outcome in firstepisode schizophrenia. Am J Psychiatry 149: 1183-1188.

Malla AK, Mittal C, Lee M, Scholten DJ, Assis L, Norman RMG (2002). Computed tomography of the brain morphology of patients with first-episode schizophrenic psychosis. J Psychiatry Neurosci JPN 27: 350-358.

Marshall M, Lewis S, Lockwood A, Drake R, Jones P, Croudace T (2005). Association between duration of untreated psychosis and outcome in cohorts of first-episode patients: a systematic review. Arch Gen Psychiatry 62: 975-983.

Miller EK, Cohen JD (2001). An integrative theory of prefrontal cortex function. Annu Rev Neurosci 24: 167-202.

Minzenberg MJ, Laird AR, Thelen S, Carter CS, Glahn DC (2009). Meta-analysis of 41 functional neuroimaging studies of executive function in schizophrenia. Arch Gen Psychiatry 66: $811-822$

Müller NG, Knight RT (2006). The functional neuroanatomy of working memory: contributions of human brain lesion studies. Neuroscience 139: 51-58.

Nielsen MO, Rostrup E, Wulff S, Bak N, Broberg BV, Lublin H et al (2012). Improvement of brain reward abnormalities by antipsychotic monotherapy in schizophrenia. Arch Gen Psychiatry 69: 1195-1204.

Nielsen MØ, Rostrup E, Wulff S, Glenthøj B, Ebdrup BH (2016). Striatal reward activity and antipsychotic-associated weight change in patients with schizophrenia undergoing initial treatment. JAMA Psychiatry 73: 121-128.

Norman RM, Townsend L, Malla AK (2001). Duration of untreated psychosis and cognitive functioning in first-episode patients. $\mathrm{Br} \mathrm{J}$ Psychiatry J Ment Sci 179: 340-345.

Penttilä M, Jääskeläinen E, Hirvonen N, Isohanni M, Miettunen J (2014). Duration of untreated psychosis as predictor of long-term outcome in schizophrenia: systematic review and meta-analysis. Br J Psychiatry J Ment Sci 205: 88-94.

Perkins D, Lieberman J, Gu H, Tohen M, McEvoy J, Green A et al (2004). Predictors of antipsychotic treatment response in patients with first-episode schizophrenia, schizoaffective and schizophreniform disorders. Br J Psychiatry J Ment Sci 185: 18-24.

Perkins DO, Gu H, Boteva K, Lieberman JA (2005). Relationship between duration of untreated psychosis and outcome in firstepisode schizophrenia: a critical review and meta-analysis. Am J Psychiatry 162: 1785-1804.

Power JD, Schlaggar BL, Petersen SE (2015). Recent progress and outstanding issues in motion correction in resting state fMRI. NeuroImage 105: 536-551.

Reilly JL, Harris MSH, Keshavan MS, Sweeney JA (2005). Abnormalities in visually guided saccades suggest corticofugal dysregulation in never-treated schizophrenia. Biol Psychiatry 57: $145-154$.

Robinson DG, Gallego JA, John M, Petrides G, Hassoun Y, Zhang J-P et al (2015). A randomized comparison of aripiprazole and risperidone for the acute treatment of first-episode schizophrenia and related disorders: 3-month outcomes. Schizophr Bull 41: $1227-1236$

Robinson DG, Woerner MG, McMeniman M, Mendelowitz A, Bilder RM (2004). Symptomatic and functional recovery from a first episode of schizophrenia or schizoaffective disorder. Am J Psychiatry 161: 473-479.

Rund BR (2014). Does active psychosis cause neurobiological pathology? A critical review of the neurotoxicity hypothesis. Psychol Med 44: 1577-1590.

Sarpal DK, Argyelan M, Robinson DG, Szeszko PR, Karlsgodt KH, John $\mathrm{M}$ et al (2016). Baseline Striatal functional connectivity as a predictor of response to antipsychotic drug treatment. Am J Psychiatry 173: 69-77.

Sarpal DK, Robinson DG, Lencz T, Argyelan M, Ikuta T, Karlsgodt K et al (2015). Antipsychotic treatment and functional connectivity of 
the striatum in first-episode schizophrenia. JAMA Psychiatry 72: $5-13$.

Seeley WW, Menon V, Schatzberg AF, Keller J, Glover GH, Kenna $\mathrm{H}$ et al (2007). Dissociable intrinsic connectivity networks for salience processing and executive control. J Neurosci 27: 2349-2356.

Srihari VH, Tek C, Pollard J, Zimmet S, Keat J, Cahill JD et al (2014). Reducing the duration of untreated psychosis and its impact in the U.S.: the STEP-ED study. BMC Psychiatry 14: 335 .
Takahashi T, Suzuki M, Tanino R, Zhou S-Y, Hagino H, Niu L et al (2007). Volume reduction of the left planum temporale gray matter associated with long duration of untreated psychosis in schizophrenia: a preliminary report. Psychiatry Res 154: 209-219.

Wyatt RJ (1991). Neuroleptics and the natural course of schizophrenia. Schizophr Bull 17: 325-351.

Yan CG, Cheung B, Kelly C, Colcombe S, Craddock RC, Di Martino A et al (2013). A comprehensive assessment of regional variation in the impact of head micromovements on functional connectomics. NeuroImage 76: 183-201.

Supplementary Information accompanies the paper on the Neuropsychopharmacology website (http://www.nature.com/npp) 